\title{
Eficiéncia Fotosintética y de Uso del Agua POR Malezas ${ }^{1}$
}

\author{
Characteristics Associated To Photosynthesis and Water Use of Weed Species
}

\author{
ASPIAZÜ, I. ${ }^{2}$, SEDIYAMA, T. ${ }^{3}$, RIBEIRO JR., J.I. ${ }^{4}$, SILVA, A.A. ${ }^{3}$, CONCENCO, G. ${ }^{5}$, GALON, L. ${ }^{6}$, \\ FERREIRA, E.A. ${ }^{7}$, SILVA, A.F. ${ }^{8}$, BORGES, E.T. ${ }^{9}$ y ARAUJO, W.F. ${ }^{10}$
}

\begin{abstract}
RESUMEN - E1 objetivo de este trabajo fue evaluar las características asociadas con la eficiencia fotosintética y del uso del agua en especies de malezas. Los tratamientos fueron compuestos por las especies Bidens pilosa, Commelina benghalensis y Brachiaria plantaginea, sembradas individualmente en las unidades experimentales. El diseño experimental fue en bloques al azar en una factorial $3 \times 4$ con tres repeticiones. Se evaluaron caracteristicas asociadas con el uso del agua - tasa transpiratoria (E), conductancia estomática (Gs), presión de vapor en la cavidad subestomatal $\left(E_{a n}\right)$ y la eficiencia del uso del agua (EUA) - asî como la fotosintesis - concentración interna de $\mathrm{CO}_{2}$ en la hoja $\left(\mathrm{C}_{\mathrm{i}}\right), \mathrm{CO}_{2}$ consumido durante la evaluación $(\Delta \mathrm{C})$, temperatura media de las hojas en el momento de la evaluación ( $\left.\mathrm{T}_{\text {leaf }}\right) \mathrm{y}$ la tasa fotosintética (A). B. plantaginea presentó menor E, menor EUA y más altos A y Ci, diferiendo de las otras especies. B. pilosa mostró valores más bajos de EUA, A y T en comparación con otras especies. Según los resultados, se puede concluir que la B. plantaginea fue más eficiente en las características relacionadas con la fotosintesis, es decir, más eficiente en el aprovechamiento de la luz. B. pilosa fue más eficiente en las características relacionadas con el uso del agua.
\end{abstract}

Palavras-clave: Bidens pilosa, Commelina benghalensis, Brachiaria plantaginea, competencia.

ABSTRACT - The objective of this study was to evaluate characteristics associated with the photosynthetic efficiency and water use of weed species. Treatments were composed by the species Bidens pilosa, Commelina benghalensis and Brachiaria plantaginea, sown individually in the experimental units. The experimental design was in randomized blocks in a $3 \times 4$ factorial, with three repetitions. Characteristics associated with water use - transpiratory rate $(E)$, stomatal conductance (Gs), vapor pressure in the substomatal chamber $\left(E_{a n}\right)$ and water use efficiency (WUE) - as well as photosynthesis - internal leaf $\mathrm{CO}_{2}$ concentration (Ci), $\mathrm{CO}_{2}$ consumed during the evaluation $(\triangle C)$, leaves average temperature at evaluation time $\left(T_{\text {leaf }}\right)$ and photosynthetic rate (A) - were evaluated. B. plantaginea showed lower E, lower WUE and higher A and Ci, differing from the other involved species. B. pilosa showed lower values of WUE, A and Twhen compared to the other species. According to the results, it can be concluded that B. plantaginea was more efficient in characteristics related to the photosynthesis, i.e., more efficient use of light. B. pilosa was more efficient in characteristics related to water use.

Keywords: Bidens pilosa, Commelina benghalensis, Brachiaria plantaginea, competition.

1 Recebido para publicação em 30.6.2009 e na forma revisada em 12.3.2010.

2 Engo-Agr ${ }^{\circ}$., D.Sc., Professor da Universidade Estadual de Montes Claros - UNIMONTES, Janaúba-MG, <aspiazu@gmail.com>;

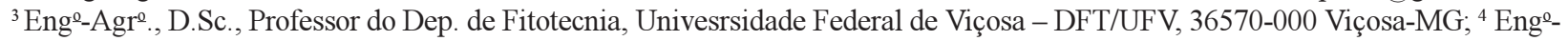
Agr $^{\circ}$., D.Sc., Prof. do Dep. de Estatística, DPE/UFV; ${ }^{5}$ Engo-Agr ${ }^{\circ}$., D.Sc., Vamont Industries, Inc. USA. Valmont Industries Irrigation Division, 7002 North 288th Street, P.O. Box 358, Valley, NE 68064 USA; ${ }^{6}$ Eng$^{0}$-Agr ${ }^{\circ}$., D.Sc., Professor da Faculdade da Amazônia - FAMA, Vilhena-RO; ${ }^{7}$ Engo-Agr ${ }^{\circ}$., D. Sc., Bolsista PNPD Universidade Federal dos Vales do Jequitinhonha e Mucuri UFVJM, Diamantina-MG; ${ }^{8}$ Eng $^{-}-$Agr $^{0}$., D.Sc., Bolsista de Pós-Doutorado, DFT/UFV; ${ }^{9}$ Estudante de graduação do curso de agronomia, UFV, bolsista iniciação científica; ${ }^{10}$ Engo-Agr $^{\circ}$., D. Sc., Prof. da Universidade Federal de Roraima - UFRR, Boa Vista-RR.

Planta Daninha, Viçosa-MG, v. 28, n. 1, p. 87-92, 2010 


\section{INTRODUCCIÓN}

Cuando las plantas son sometidas a competencia, las características fisiológicas de crecimiento y desarrollo normalmente son alteradas, lo que resulta en diferencias en la utilización de los recursos ambientales, especialmente en el uso del agua, que afecta directamente la disponibilidad de $\mathrm{CO}_{2}$ y la temperatura en el mesofilo de la hoja, consecuentemente, la eficiencia fotosintética de la planta (Loreto \& Bongi, 1989).

La eficiencia del uso del agua se define por la relación entre los gramos del agua transpirados por un cultivo, por gramo de materia seca producida. De esta manera, las culturas más eficientes en el uso del agua producen más materia seca por gramo del agua transpirado. En plantas que presentan metabolismo $\mathrm{C}_{3} \circ \mathrm{C}_{4}$, el uso más eficiente del agua está directamente correlacionado con el tiempo de apertura estomática y resistencia estomática, ya que mientras la planta absorbe el $\mathrm{CO}_{2}$ para la fotosintesis, el agua se pierde por transpiración, con intensidad variable en función de la conductancia estomática y del gradiente de potencial entre la superficie foliar y la atmósfera, siguiendo una corriente de potenciales hídricos (Pereira-Netto et al., 2002).

Varios son los factores que influyen directa o indirectamente en la fotosintesis; déficit hídrico, estrés térmico (Loreto \& Bongi, 1989), concentración interna y externa de gases (Kirschbaum \& Pearcey, 1988) y composición e intensidad de la luz (Sharkey \& Raschke, 1981). Aunque la capacidad de conducción e intercambio gaseoso por estomas se considera como la principal limitación de la asimilación de $\mathrm{CO}_{2}$ para el proceso fotosintético (Hutmacher \& Krieg, 1983), es poco probable que el intercambio de los gases por si solo pueda limitar la tasa de fotosintesis cuando en interacción con otros factores. La tasa fotosintética esta directamente relacionada con la radiación fotosinteticamente activa (composición de la luz), a los factores de disponibilidad del agua, y al intercambio de los gases (Naves-Barbiero et al., 2000).

El objetivo de este trabajo fue evaluar las características asociadas a la eficiencia fotosintética y de uso del agua de las malezas
Bidens pilosa, Commelina benghalensis y Brachiaria plantaginea.

\section{MATERIAL Y MÉTODOS}

E1 experimento se llevó a cabo en un ambiente abierto al final de la temporada de lluvias, en la Universidad Federal de Viçosa, en el campo experimental Diogo Alves de Mello. Las unidades experimentales consistieron de recipientes de fibra de vidrio con una capacidad de $150 \mathrm{dm}^{3}$, rellenos con el suelo clasificado como Haplustox, previamente fertilizado según Novais et al. (2007).

Los tratamientos fueron compuestos por las especies Bidens pilosa, Commelina benghalensis y Brachiaria plantaginea cultivadas individualmente en las unidades experimentales. Fueron sembradas en cada parcela experimental, tal como el tratamiento especificado, las malezas Bidens pilosa (3 plantas $\left.\mathrm{m}^{-2}\right)$ o Brachiaria plantaginea (6 plantas $\mathrm{m}^{-2}$ ), donde la Commelina benghalensis trasplantada por plantones (3 plantas $\mathrm{m}^{-2}$ ). Durante todo el período de realización del experimento se mantuvo la humedad del suelo en torno a $2 / 3$ de capacidad de campo por medio riego regular de acuerdo con la necesidad.

A los 60 días después de la emergencia de Bidens pilosa y Brachiaria plantaginea y trasplante de Commelina benghalensis, las evaluaciones se llevaron a cabo en las tres especies con un analizador de los gases infrarrojo (IRGA), modelo ADC-LCA 4 (Analytical Development Co. Ltd., Hoddesdon, Herts, UK), entre 8 y $10 \mathrm{~h}$ de la mañana, siendo evaluadas las características asociadas con el uso del agua - tasa transpiratória (E $\mathrm{H}_{2} \mathrm{O} \mathrm{mol} \mathrm{m} \mathrm{m}^{-2} \mathbf{s}^{-1}$, conductancia estomática $\left(\mathrm{Gs}-\mathrm{mol} \mathrm{m}^{-1} \mathrm{~s}^{-1}\right)$, presión de vapor en la cavidad subestomatal ( $\left.E_{\text {an }}-m b a r\right)$, eficiencia del uso del agua (EUA - mol de $\mathrm{CO}_{2} \mathrm{~mol} \mathrm{H}_{2} \mathrm{O}^{-1}$ ) , bien como las asociadas a la fotosintesis concentración interna de $\mathrm{CO}_{2}$ en la hoja (Ci mol mol ${ }^{1}$ ), $\mathrm{CO}_{2}$ consumido durante la evaluación $\left(\Delta \mathrm{C}-\mathrm{mol} \mathrm{mol}{ }^{-1}\right)$, temperatura media de las hojas en el momento de la evaluación $\left(\mathrm{T}_{\text {leaf }}-{ }^{\circ} \mathrm{C}\right)$ y tasa fotosintética (A $\mathrm{mol} \mathrm{m} \mathrm{m}^{-2} \mathbf{s}^{-1}$ ). Las evaluaciones se llevaron a cabo en el tercio medio de la primera hoja completamente expandida del vástago 
principal de $B$. plantaginea, en el trifolio central de la primera hoja completamente expandida de $B$. pilosa, y en la primera hoja completamente expandida de $C$. benghalensis.

Los datos fueron sometidos a análisis de varianza por el test $\mathrm{F}$ y, en caso de significancia, se compararon las medias a los pares $(\mathrm{p}<0.05)$ por el test de la minima diferencia significativa (LSD). Todos las análisis fueron ejecutadas en el software estadístico SAEG.

\section{RESULTADOS Y DISCUSIÓN}

La conductancia foliar (Gs) no fue diferente $(P>0,05)$ entre las especies evaluadas, con valores alrededor de $0,06 \mathrm{~mol} \mathrm{~m}^{-1} \mathbf{s}^{-1}$. La conductancia foliar es compuesta en parte por la pequeña conductancia cuticular de la epidermis y, cuando los estomas están abiertos, ocurre control de esta por sus células guarda. Por eso, la Gs es proporcional al número y tamaño de estomas y a su diámetro de apertura, características que dependen de otros factores endógenos y ambientales (Brodribb \& Holbrook, 2003). La capacidad de intercambio de vapor del agua entre las superficies interior y exterior de la hoja fue similar para todas las especies evaluadas (Tabla 1). El control de apertura y el cierre de estomas depende de varios factores tales como radiación solar, niveles de $\mathrm{CO}_{2}$ en el mesófilo, humedad relativa (déficit de presión de vapor del aire), potencial hídrico, y otros de menor magnitud como el viento, sustancias de crecimiento y ritmos endógenos propios de cada especie.

De manera similar a la Gs, la $\mathrm{E}_{\text {an }}$ tampoco fue alterada $(P>0,05)$ en función de la especie evaluada. La $\mathrm{E}_{\text {an }}$ está directamente relacionada al estatus hídrico de la planta y a la dinámica del flujo de vapor del agua. Mismo en una hoja con alta tasa transpiratória, la humedad relativa en la cavidad subestomatal podrá superar un $95 \%$ y el potencial hídrico resultante puede estar cerca de un $0 \%$ (cero), incrementando los intercambios con el ambiente, que tiene potencial hídrico muy negativo. Bajo estas condiciones, la presión de vapor es la presión de vapor de la saturación a la temperatura de la hoja. De esta forma, la $\mathrm{E}_{\mathrm{an}}$ es controlada por el nivel de humedad y temperatura de la hoja, y actúa sobre la conductancia estomática de vapor del agua (Gs) y la transpiración (E).

Tabla 1 - Variables asociadas a la fotosíntesis e al uso del agua, en función de la especie de maleza

\begin{tabular}{|c|c|c|c|c|}
\hline \multicolumn{5}{|c|}{ Uso del agua* } \\
\hline Especie Maleza & $\begin{array}{c}\text { Gs } \\
\left(\mathrm{mol} \mathrm{m}^{-1} \mathrm{~s}^{-1}\right)\end{array}$ & $\begin{array}{c}\mathrm{E}_{\mathrm{an}} \\
(\mathrm{mBar})\end{array}$ & $\begin{array}{c}\mathrm{E} \\
\left(\mathrm{mol} \mathrm{H}_{2} \mathrm{O} \mathrm{m}^{-2} \mathrm{~s}^{-1}\right)\end{array}$ & $\begin{array}{c}\text { EUA } \\
\left.\mathrm{mol} \mathrm{CO}_{2} \mathrm{~mol} \mathrm{H}_{2} \mathrm{O}^{-1}\right)\end{array}$ \\
\hline Bidens pilosa & $0,08 \mathrm{a}^{1 /}$ & $19,90 \mathrm{a}$ & $2,17 \mathrm{a}$ & $0,98 \mathrm{~b}$ \\
\hline Commelina benghalensis & $0,07 \mathrm{a}$ & $21,57 \mathrm{a}$ & $2,40 \mathrm{a}$ & $1,26 \mathrm{~b}$ \\
\hline Brachiaria plantaginea & $0,04 \mathrm{a}$ & $20,30 \mathrm{a}$ & $1,71 \mathrm{~b}$ & $4,85 \mathrm{a}$ \\
\hline CV $(\%)$ & 32,9 & 4,2 & 16,8 & 91,3 \\
\hline \multicolumn{5}{|c|}{ Fotosíntesis* } \\
\hline Espécie Maleza & $\begin{array}{c}\mathrm{C}_{\mathrm{i}} \\
\left(\mathrm{mol} \mathrm{mol}^{-1}\right)\end{array}$ & $\begin{array}{c}\mathrm{C} \\
\left(\mathrm{mol} \mathrm{mol}^{-1}\right)\end{array}$ & $\begin{array}{l}\mathrm{T}_{\text {leaf }} \\
\left({ }^{\circ} \mathrm{C}\right)\end{array}$ & $\begin{array}{c}\mathrm{A} \\
\left(\mathrm{mol} \mathrm{m}^{-2} \mathrm{~s}^{-1}\right)\end{array}$ \\
\hline Bidens pilosa & $303,6 \mathrm{a}$ & $8,30 \mathrm{~b}$ & $30,9 \mathrm{~b}$ & $2,26 \mathrm{~b}$ \\
\hline Commelina. benghalensis & $282,1 \mathrm{~b}$ & $8,17 \mathrm{~b}$ & $34,3 \mathrm{a}$ & $3,07 \mathrm{~b}$ \\
\hline Brachiaria plantaginea & $31,2 \mathrm{c}$ & $21,83 \mathrm{a}$ & $35,1 \mathrm{a}$ & $8,44 \mathrm{a}$ \\
\hline $\mathrm{CV}(\%)$ & 73,6 & 61,5 & 7,4 & 73,2 \\
\hline
\end{tabular}

1/ Medias seguidas por la misma letra, en la columna, no difieren entre si por el test LSD $(\mathrm{P}>0,05)$. ${ }^{*}$ Conductancia estomática de vapores de agua (Gs), presión de vapor en la cavidad subestomática $\left(\mathrm{E}_{\text {an }}\right)$, tasa transpiratória (E), efíciencia del uso del agua (EUA), concentración interna de $\mathrm{CO}_{2}$ en la hoja $(\mathrm{Ci}), \mathrm{CO}_{2}$ consumido $(\Delta \mathrm{C})$, temperatura de la hoja $\left(\mathrm{T}_{\text {leaf }}\right)$ y tasa fotosintética $(\mathrm{A})$. 
La especie B. pilosa es ampliamente conocida por su alta capacidad de extracción del agua del suelo, aunque no sea muy eficiente en el uso de la misma. Esta especie es capaz de mantener su crecimiento en valores de potencial hídrico en que la mayoria de las malezas atingió el punto de marchitamiento permanente, y, en algunos casos, se beneficia por la ocurrencia de estrés hídrico moderado (Procópio, 2004). La especie $B$. plantaginea, por otro lado, tiene distinta estrategia de $B$. pilosa para sobrevivir bajo el estrés hídrico. Mientras $B$. pilosa es muy eficiente en la extracción del recurso, B. plantaginea es eficiente en su uso, por presentar fisiología $\mathrm{C}_{4}$ del metabolismo del carbono. Como B. plantaginea es capaz de continuar incorporando carbono mismo en concentraciones cerca de un cero ppm, puede pasar más tiempo con los estomas cerrados y aumentar la eficiencia del uso del recurso agua.

La disminución de la transpiración (E) está asociada al cierre de los estomas, y cambios en la apertura estomática causan alteraciones potenciales en el potencial hídrico, por actuar sobre la E (Brodribb \& Hill, 2000). La planta tiende a cerrar sus estomas cuando los niveles de luz están bajo la radiación fotosintéticamente activa, o para evitar el estrés hídrico (Cochard et al., 2002). La planta de $B$. plantaginea fue superior $(\mathrm{P}<0,05)$ a las demás cerca la pérdida del agua por transpiración. Presumiblemente, esto se debe al hecho de esta planta presentar metabolismo $\mathrm{C}_{4}$, y tener más bajo punto de compensación de $\mathrm{CO}_{2}$. Luego, es capaz de reducir la apertura de los estomas por mayor período de tiempo, lo que resulta en reducción de la pérdida del agua por transpiración. Las características $\mathrm{Gs}, \mathrm{E}_{\text {an }}, \mathrm{y}$ E están vinculadas en una relación costo-beneficio, pues los procesos de transpiración y de captura de $\mathrm{CO}_{2}$ sólo ocurren cuando los estomas están abiertos, bien como la Gs. Además, $E_{a n}$ normalmente es muy reducida en los períodos de apertura estomática.

La eficiencia del uso del agua (EUA) representa la cantidad de $\mathrm{CO}_{2}$ fijado para la producción de materia seca en función de la cantidad del agua transpirada. La mayor EUA $(\mathrm{P}<0,05)$ observada para $B$. plantaginea sobre las demás especies está directamente relacionada a la menor transpiración observada para esta especie, ya que, como las plantas $\mathrm{C}_{4}$ son capaces de capturar el $\mathrm{CO}_{2}$ bajo menores concentraciones en el mesófilo que las plantas $\mathrm{C} 3$, pasan menos tiempo con estomas abiertos (Tabla 1).

La Ci de la hoja diferio entre especies, de las que la $B$. pilosa mostró mayores $(\mathrm{P}<0,05)$ niveles de $\mathrm{CO}_{2}$, seguida de $C$. benghalensis y B. plantaginea (Tabla 1). Esta es considerada una característica fisiológica influenciada por factores ambientales como la disponibilidad del agua, de la luz y de la energía, entre otros (Ometto et al., 2003). Cuanto más alta sea la tasa fotosintética de las especies, más rápido se consume el $\mathrm{CO}_{2}$, y menor es su concentración en el interior de la hoja, suponiéndose que los estomas estén cerrados (Corniani et al., 2006). El consumo de $\mathrm{CO}_{2}$ aumenta las diferencias en la concentración de este gas entre el interior de la hoja y el ambiente externo, y que, por lo general, cuanto mayor sea el gradiente $(\Delta \mathrm{C})$, más rápidamente el $\mathrm{CO}_{2}$ entrará en la hoja después de la apertura estomática en función de un mayor gradiente de concentración. Por eso, se constató también que la menor concentración interna de $\mathrm{CO}_{2}$ (Ci) ocasionó mayor gradiente de gas entre el interior y el exterior de la hoja $(\Delta \mathrm{C})$.

En función del metabolismo de la planta, la temperatura de la hoja es, por lo general, superior a la del aire a su alrededor. Así, los cambios en el metabolismo pueden ser medidos indirectamente en función de la temperatura de la hoja mientras el mismo permanezca activo (Atkin et al., 2000). De acuerdo con lo visto para $\mathrm{Ci}$ y $\Delta \mathrm{C}$, la temperatura de la hoja fue menor $(\mathrm{P}<0,05)$ para B. pilosa, pues esta mostró un menor consumo de $\mathrm{CO}_{2}$ que las demás especies, con mayor $\mathrm{Ci}$ y menor $\Delta \mathrm{C}$ que $B$. plantaginea (Tabla 1).

Similarmente a lo encontrado para las variables $\mathrm{Ci}, \Delta \mathrm{C}$ y $\mathrm{T}_{\text {leaf }}$, la fotosintesis fue mayor para $B$. plantaginea en comparación con otras especies (Tabla 1), probablemente en función de que esta presenta metabolismo $\mathrm{C}_{4}$, con un menor punto de saturación de $\mathrm{CO}_{2}$, mayor punto de compensación luminosa y mayor eficiencia del uso del agua que las plantas $\mathrm{C}_{3}$ 
(Taiz \& Zeiger, 2006). La fotosintesis, y consecuentemente la respiración, dependen del flujo constante de $\mathrm{CO}_{2}$ y $\mathrm{O}_{2}$ entrando y saliendo de la célula; este libre flujo ocurre en función de la concentración de esos gases en los espacios intercelulares dependientes de la apertura estomática, controladora mayoritaria del flujo de estos (Messinger et al., 2006). Esta, a su vez, es en gran parte controlada por la turgescencia tanto de las células de guarda (que controlan la apertura de los estomas) como de las células epidérmicas de los estomas (Taiz \& Zeiger, 2006). Un bajo potencial hídrico, que induce el cierre de los estomas y reduce la conductancia foliar, inhibe la fotosintesis y también la respiración.

La luz afecta la apertura estomática indirectamente por su efecto en la asimilación de $\mathrm{CO}_{2}$ (Sharkey \& Raschke, 1981; Nishio et al., 1994). Sin embargo, la apertura estomática es menos dependiente de la $\mathrm{Ci}$, respondiendo directamente a la luz (Radosevich, 1997). Así pues, en condiciones de competencia y sombreamiento, el balance de la luz que participa en el control de la apertura estomática y en el balance de los gases entre el interior de la hoja y el ambiente externo (Loreto \& Bongi, 1989; Tallman \& Zeiger, 1988). En estudios con plantas de girasol sometidas a estrés hídrico, la concentración de $\mathrm{CO}_{2}$ dentro de la hoja (Ci) aumentó, mientras que la tasa de fotosintesis (A) se redujo. Al quitar el agente causante del estrés, la planta recuperó la capacidad fotosintética y normalizó el Ci (Corniani et al., 2006). Los mismos resultados se obtuvieron en este trabajo en función de las especies evaluadas (Tabla 1).

Por lo general, es posible inferir que, en condiciones de menor disponibilidad hídrica, $B$. plantaginea tiende a sobrepasar $B$. pilosa y $C$. benghalensis en relación con la competitividad debido a su menor tasa transpiratória, alta eficiencia en el uso del agua, metabolismo más acurado (medido por la temperatura de la hoja, menor Ci y mayor $\Delta$ C) y mayor tasa fotosintética. Sin embargo, en condiciones de estrés hídrico, B. pilosa puede ganar la competencia, ya que esta especie puede extraer el agua del suelo en condiciones de muy bajo potencial, lo que no ocurre con B. plantaginea. Esta, a su vez, fue inferior a C. benghalensis cuanto al consumo de $\mathrm{CO}_{2} \mathrm{y}$ temperatura de la hoja, lo que indica menor tasa metabólica. No obstante, esta especie puede tener ventajas sobre $C$. benghalensis, principalmente en función de la alta capacidad para extraer el agua del suelo, lo que puede compensar, al menos en parte, su menor eficiencia en el uso de este recurso.

\section{LITERATURA CITADA}

ATKIN, O. K. et al. Leaf respiration of Snow Gum in the light and dark. Interactions between temperature and irradiance.

Plant Physiol., v. 122, n. 3, p. 915-923, 2000.

BRODRIBB, T. J.; HOLBROOK, N. M. Stomatal closure during leaf dehydration, correlation with other leaf physiological traits. Plant Physiol., v. 132, n. 4, p. 2166-2173, 2003.

COCHARD, H. et al. Unraveling the effects of plant hydraulics on stomatal closure during water stress in walnut. Plant Physiol., v. 128, p. 282-290, 2002.

CORNIANI, N. et al. Determinação das trocas gasosas e de potencial hídrico através do uso de sistemas portáteis na avaliação do estresse. In: SIMPÓSIO INTERNACIONAL DE INICIAÇÃO CIENTÍFICA DA UNIVERSIDADE DE SÃO PAULO, 14., 2006, Piracicaba. Anais... São Paulo: USP, 2006. CD ROM.

HUTMACHER, R. B.; KRIEG, D. R. Photosynthetic rate control in cotton. Plant Physiol., v. 73, n. 3, p. 658-661, 1983.

KIRSCHBAUM, M. U. F.; PEARCY, R. W. Gas exchange analysis of the relative importance of stomatal and biochemical factors in phosynthetic induction in Alocasia macrorrhiza. Plant Physiol., v. 86, n. 3, p. 782-785, 1988.

LORETO, F.; BONGI, G. Combined low temperature-high light effects on gas exchange properties of jojoba leaves. Plant Physiol., v. 91, n. 4, p. 1580-1585, 1989.

MESSINGER, S. M. et al. Evidence for involvement of photosynthetic processes in the stomatal response to $\mathrm{CO}_{2}$. Plant Physiol., v. 140, n. 2, p. 771-778, 2006.

NAVES-BARBIERO, C. C. et al. Fluxo de seiva e condutância estomática de duas espécies lenhosas sempre-verdes no campo sujo e cerradão. R. Bras. Fisiol. Veg., v. 12, n. 1, p. 119-134, 2000 .

NISHIO, J. N.; SUN, J.; VOGELMANN, T. C. Photoinhibition and the light environment within leaves. In: BAKER, N. R.; BOWYER, J. R. (Eds.). Photoinhibition of photosynthesis. Oxford: BIOS Scientific Publishers, 1994. p. 1-24.

NOVAIS, R. F. et al. Fertilidade do solo. Viçosa-MG: Sociedade Brasileira de Ciência do Solo, 2007. 1017 p. 
OMETTO, J. P. H. B. et al. Variação temporal do isótopo estável do carbono em material arbóreo em florestas da região Amazônica. In: CONGRESSO BRASILEIRO DE ECOLOGIA, 4., 2003, Fortaleza. Anais... Rio Claro: Sociedade de Ecologia do Brasil, 2003. CD ROM

PEREIRA-NETTO, A. B. Crescimento e desenvolvimento. In: WACHOWICZ, C. M.; CARVALHO, R. I. N. (Eds.) Fisiologia vegetal - produção e pós-colheita. Curitiba: Champagnat, 2002. p. 17-42

PROCÓPIO, S. O. et al. Características fisiológicas das culturas de soja e feijão e de três espécies de plantas daninhas Planta Daninha, v. 22, n. 2, p. 211-216, 2004.
RADOSEVICH, S. R.; HOLT, J.; GHERSA, C. Weed ecology: implication for managements. New York: John Willey \& Sons, 1997. 589 p.

SHARKEY, T. D.; RASCHKE, K. Effect of light quality on stomatal opening in leaves of Xanthium strumarium L. Plant Physiol., v. 68, n. 5, p. 1170-1174, 1981.

TAIZ, L.; ZEIGER, E. Plant physiology. Sunderland: Sinauer, 2006. 705 p.

TALLMAN, G.; ZEIGER, E. Light quality and osmoregulation in Vicia guard cells. Plant Physiol., v. 88, p. $887-895,1988$. 